\title{
Open cluster remnants in low-density Galactic fields
}

\author{
D. B. Pavani, ${ }^{1}$ L. O. Kerber,${ }^{2}$ E. Bica ${ }^{3}$ and W. J. Maciel ${ }^{4}$ \\ ${ }^{1}$ Universidade Federal de Pelotas (UFPel), Campus Universitário, s/n, Caixa Postal 354, \\ CEP 96010-900 Pelotas, RS, Brazil \\ email: dpavani@if.ufrgs.br \\ ${ }^{2}$ Universidade Estadual de Santa Cruz (UESC), Campus Soane Nazaré de Andrade km 16 \\ Rodovia Ilhéus-Itabuna CEP 45662-000, Ilhéus, Bahia, Brazil \\ e-mail: [kerber, maciel] @astro.iag.usp.br \\ ${ }^{3}$ Universidade Federal do Rio Grande do Sul (UFRGS), Av. Bento Goncalves 9500, \\ Caixa Postal 15051, CEP 91501-970 Porto Alegre, RS, Brazil \\ email: bica@if.ufrgs.br \\ ${ }^{4}$ Instituto de Astronomia, Geofísica e Ciências Atmosféricas da Universidade de São Paulo \\ (IAG/USP), Rua do Matão, 1226, Cidade Universitária, CEP 05508-090 São Paulo/SP, Brazil
}

\begin{abstract}
Open cluster remnants (OCRs) are fundamental objects to investigate open cluster dissolution processes (e.g., Bica et al. 2001; Carraro 2002; Pavani et al. 2003; Carraro et al. 2007; Pavani \& Bica 2007). They are defined as poorly populated concentrations of stars, with enough members to show evolutionary sequences in colour-magnitude diagrams (CMDs) as a result of the dynamical evolution of an initially more massive physical system. An OCR is intrinsically poorly populated, which makes its differentiation from field-star fluctuations difficult. Among the possible approaches to establish the nature of OCRs, we adopted CMD analysis combined with a robust statistical tool applied to 2MAss data. In addition, photometry is the main information source available for possible OCRs (POCRs). We developed a statistical diagnostic tool to analyse the CMDs of POCRs and verify them as physical systems, explore membership probabilityies taking into account field contamination and derive age, distance and reddening values in a self-consistent way. We present the results of our analysis of 88 POCRs that are part of a larger sample that is widely distributed across the sky, with a significant density contrast of bright stars compared to the Galactic field. The 88 objects are projected onto low-density Galactic fields, at relatively high latitudes $\left(|b|>15^{\circ}\right)$. Studies of larger POCR samples will provide a better understanding of OCR properties and constraints for theoretical models, including new insights into the evolution of open clusters and their dissolution rates. The results of this ongoing survey will provide a general picture of these fossil stellar systems and their connection to Galactic-disk evolution.
\end{abstract}

Keywords. open clusters and associations: general, infrared: general, methods: statistical

\section{The sample}

The objects were selected from open cluster catalogues and visual inspection of Digitised Sky Survey maps. All objects are underpopulated compared with the 'usual' open clusters, so that availability of proper-motion, radial-velocity or spectral studies is rare. However, photometry is the main information source available for POCRs and the 2MASS database provides objects and fields in a homogeneous manner, together with photometric errors and completeness estimates. Figure 1 shows an overall view of the complete sample. The sample contains 36 objects selected from the NGC catalogue, 32 from the ESO-LV, 7 from Ruprecht, 5 from IC, 5 from Collinder and 3 from elsewhere. 


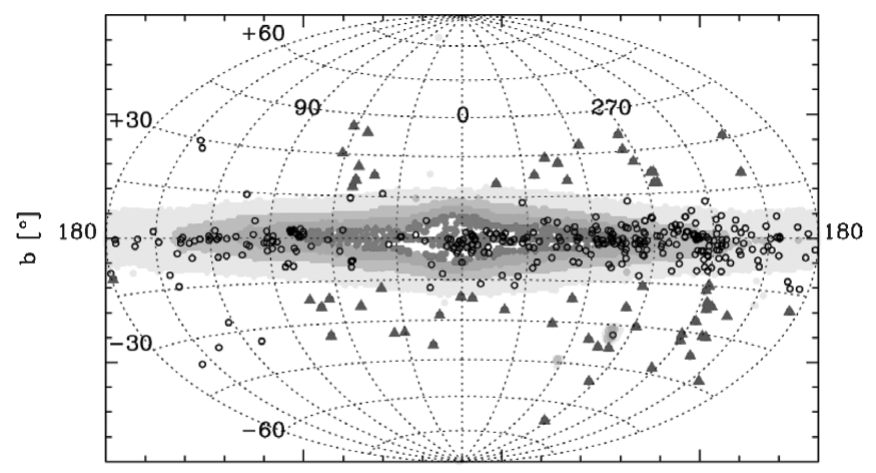

$1\left[^{\circ}\right]$

Figure 1. Whole-sky Aitoff projection showing, in Galactic coordinates, the positions of the present POCR sample (triangles) and the larger sample of POCRS (open circles). 2MAss background stellar densities are shown at four levels, corresponding to the 20th, 40th, 60th and 80th percentiles of the $\log \left(\rho_{\text {field }}\right)$ distribution.
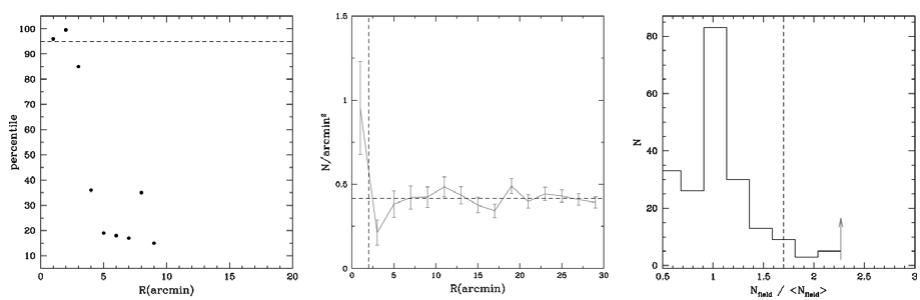

Figure 2. (left) Example of the search for the largest radius that maximizes the number of stars in the POCR compared with the counts in the field-star distribution (percentiles). The horizontal dashed line indicates the 95th percentile. (middle) Example of radial stellar density profiles compared with the stellar-field density averages (horizontal dashed line). $R_{\text {cut }}$ is also shown (vertical dashed line). (right) Example of star-count distributions for the field-star samples with the same radius as the object, compared with the POCR ( $N_{\mathrm{POCR}}$; arrow).

\section{Method}

The method assigns to each star a membership probability and finds a set of best isochrone fits, maximizing the number of fitted stars. Applying the same method to the randomly selected neighbouring fields, it objectively tests whether or not POCRs can be understood as field fluctuations.

(a) Determining the POCR cutoff radius. This corresponds to star-count analysis of the POCR sample $\left(N_{\mathrm{POCR}}\right)$ compared with the field $\left(N_{\text {field }}\right)$. We use neighbouringfield samples randomly selected from concentric background regions as control fields. The number of comparison fields depends on the ratio of the field-to-POCR areas. The process to determine the cutoff radius $\left(R_{\text {cut }}\right)$ is shown in Figure 2.

(b) Taking into account field-star contamination. This method was inspired by the field-star decontamination process of Mighell et al. (1996), Kerber et al. (2002) and Kerber \& Santiago (2005). We adapted their central ideas and applied them to poorly populated objects. Therefore, we assume that the CMD of a control field is representative of the field. By a comparison with the POCR CMD, we compute the probability for any POCR star to be a member. We developed a semi-automated method to test a suitable number of possible isochrone solutions (for solar metallicity). First, we estimate initial 

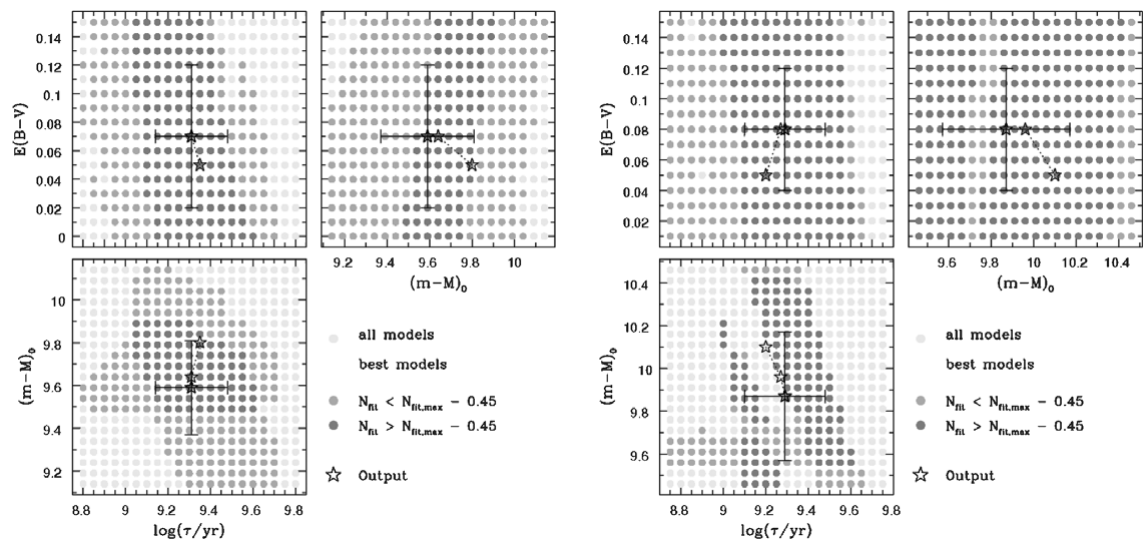

Figure 3. Example of the parameter space explored for ESO324-SC15, which was identified as an OCR (left) and NGC 272 (right), classified as a field fluctuation. The evolution of the output solution (open stars) is shown.
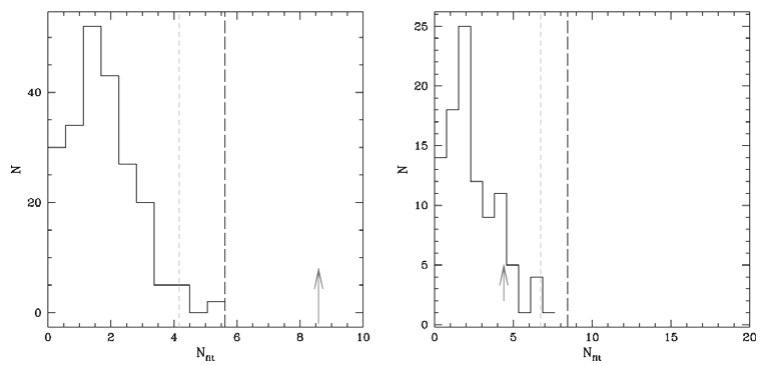

Figure 4. Examples of the distributions of the isochrone-fitted stars for the field-star samples compared with the object (arrow).

guesses for each physical parameter by eye and we subsequently explore a large parameter space in equal steps. Therefore, for each POCR we typically test 8000 isochrone solutions. The parameter space explored is shown in Figure 3. These figures show the best solutions and the evolution of the average (output) solution after each iteration.

(c) Statistical comparison with field stars. The statistical comparison with the field and the final characterization are illustrated in Figure 4, which presents the number of isochrone-fitted stars, taking into account the probability distribution for the field stars $\left(n_{\mathrm{fit}}\right)$ compared with the value for each object $\left(n_{\mathrm{fit}, \mathrm{POCR}}\right)$. The number of fitted stars for ESO324-SC15 are not reproduced by their neighbouring field samples, thus being classified as OCR by the criterion. On the other hand, the same figure also reveals that NGC 272 can be understood as a field stellar fluctuation (FF). In Figure 5 we present CMDs for two OCRs: ESO435-SC48- $\log ($ age/yr $)=9.3 \pm 0.17, E(B-V)=0.07 \pm$ $0.05 \mathrm{mag},(m-M)_{0}=9.59 \pm 0.22 \mathrm{mag}-$ and ESO324-SC15- $\log (\mathrm{age} / \mathrm{yr})=9.1 \pm 0.1$, $E(B-V)=0.08 \pm 0.05 \mathrm{mag},(m-M)_{0}=9.60 \pm 0.23 \mathrm{mag}$.

\section{Results and concluding remarks}

Figure 6 provides an overview of the properties of the OCRs. The method assigns to each star a membership probability and finds a set of best isochrone fits, maximizing the number of fitted stars. The method objectively tests whether or not the possible open 

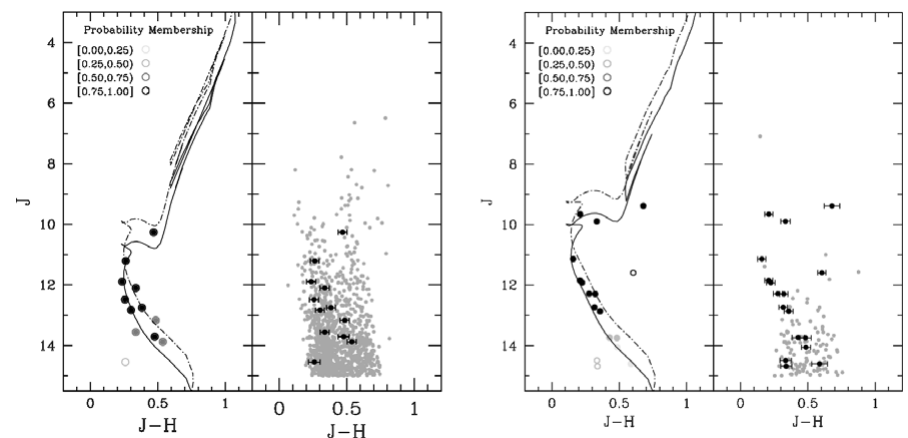

Figure 5. $J$ versus $(J-H)$ CMD of photometric OCRs: ESO435-SC48 (left) and ESO324-SC15 (right) according to their stellar probability membership. Padova isochrones (Girardi et al. 2002) of solar metallicity are shown as well as a shifted version including binaries of equal masses (dashed line). (right) Cluster stars overplotted on the stars in the control field $\left(R=30^{\prime}\right)$.

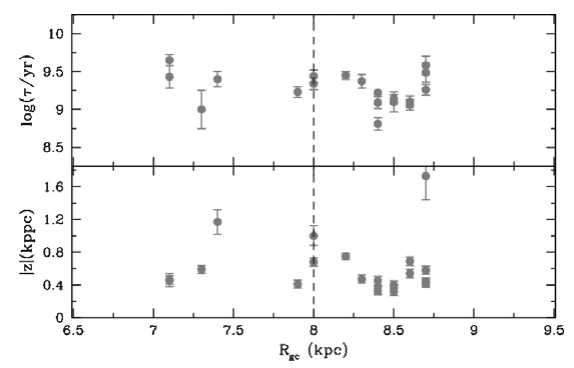

Figure 6. The objects are shown as a function of height from the plane $|z|$ and age, as a function of Galactocentric distance $R_{\mathrm{gc}}$ (right). The Sun is assumed to be at $R_{\mathrm{gc}}=8 \mathrm{kpc}$ (Reid 1993).

cluster remnant can be understood as a field fluctuation. The objective criteria suggested that 23 objects were OCRs, 45 FFs, and 20 were classified as borderline objects. These results are consistent with their behaviour in parameter space (Figure 3 ).

\section{References}

Bica, E., Santiago, B. X., Dutra, C. M., Dottori, H., de Oliveira, M. R., \& Pavani, D. 2001, $A \mathscr{G} A, 366,827$

Carraro, G. 2002, A\&A, 385, 471

Carraro, G., de La Fuente Marcos, R., Villanova, S., Moni Bidin, C., de La Fuente Marcos, C., Solivella, G., \& Baumgardt, H. 2007, A\&A, 466, 931

Girardi, L., Bertelli, G., Bressan, A., Chiosi, C., Groenewegen, M. A. T. Marigo, P., Salasnich, B., \& Weiss, A. 2002, A\&A, 391, 185

Kerber, L. O, Santiago, B. X., Castro, R., \& Valls-Gabaud, D. 2002, A\&\&A, 390, 121

Kerber, L. O. \& Santiago, B. X. 2005, A\&A A, 435, 77

Miguell, K. J., Rich, R. M., Shara, M., \& Fall, S. M. 1996, A\& A, 111, 2314

Pavani, D. B., Bica, E., Ahumada, A. V., \& Clariá, J. J. 2003, A\&\&A, 399, 113

Pavani, D. B. \& Bica, E. 2007, A\&SA, 468, 139

Reid, M. J. 1993, ARA\&A, 31345 